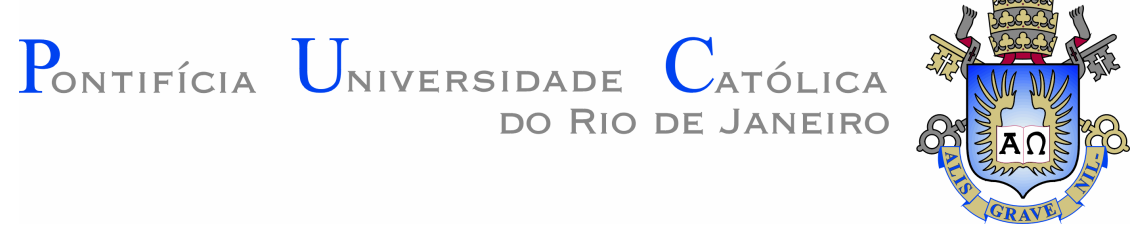

Otávio da Fonseca Martins Gomes

Microscopia Co-Localizada:

Novas Possibilidades na Caracterização de Minérios

Tese de Doutorado

Tese apresentada como requisito parcial para obtenção do grau de Doutor em Engenharia Metalúrgica e de Materiais pelo Programa de PósGraduação em Engenharia Metalúrgica do Departamento de Ciência dos Materiais e Metalurgia da PUC-Rio.

Orientador: Prof. Sidnei Paciornik 


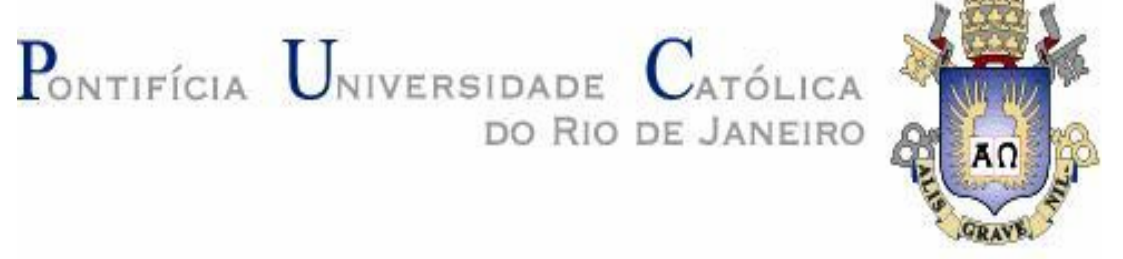

OTÁVIO DA FONSECA MARTINS GOMES

\section{Microscopia Co-Localizada: Novas Possibilidades na Caracterização de Minérios}

Tese apresentada como requisito parcial para obtenção do grau de Doutor em Engenharia Metalúrgica e de Materiais pelo Programa de Pós-Graduação em Engenharia Metalúrgica do Departamento de Ciência dos Materiais e Metalurgia da PUC-Rio. Aprovada pela Comissão Examinadora abaixo assinada.

Prof. Sidnei Paciornik

Orientador

Departamento de Ciência dos Materiais e Metalurgia - PUC - Rio

Dr. Marcos Henrique de Pinho Maurício Departamento de Ciência dos Materiais e Metalurgia - PUC - Rio

Prof. Eduardo de Albuquerque Brocchi Departamento de Ciência dos Materiais e Metalurgia - PUC - Rio

Prof. José Carlos Gaspar Universidade de Brasília - UNB

Prof. Claudio Luiz Schneider Centro de Tecnologia Mineral - CETEM

Prof. José Eugenio Leal Coordenador Setorial de Pós-Graduação do Centro Técnico Científico da PUC-Rio 
Todos os direitos reservados. É proibida a reprodução total ou parcial do trabalho sem autorização da universidade, do autor e do orientador.

\section{Otávio da Fonseca Martins Gomes}

Possui graduação em Engenharia Química pela Universidade Federal do Rio de Janeiro (1998) e mestrado em Engenharia de Materiais pela Pontifícia Universidade Católica do Rio de Janeiro (2001). Atualmente é Tecnologista Pleno 2 do Centro de Tecnologia Mineral (CETEM/MCT). Tem experiência na área de Engenharia de Materiais e Metalurgia, com ênfase em Caracterização Tecnológica de Minérios e Materiais, atuando principalmente nos seguintes temas: microscopia (eletrônica, óptica e digital), processamento e análise digital de imagens e classificação automática.

Ficha Catalográfica

Gomes, Otávio da Fonseca Martins

Microscopia co-localizada: novas possibilidades na caracterização de minérios / Otávio da Fonseca Martins Gomes ; orientador: Sidnei Paciornik. - 2007. 105 f. : il. ; $30 \mathrm{~cm}$

Tese (Doutorado em Ciência dos Materiais e Metalurgia) - Pontifícia Universidade Católica do Rio de Janeiro, Rio de Janeiro, 2007. Inclui bibliografia

1. Ciência dos materiais e metalurgia Teses. 2. Microscopia digital. 3. Caracterização de minérios. 4. Análise de imagens. 5. Classificação. 7. Minério de ferro. 8. Minério de cobre. I. Paciornik, Sidnei. II. Pontifícia Universidade Católica do Rio de Janeiro. Departamento de Ciência dos Materiais e Metalurgia. III. Título.

CDD: 669 


\section{Agradecimentos}

Ao meu orientador e amigo, Sidnei, que nestes 8 anos de convivência, desde o mestrado, muito me ensinou. Creio que ele também tenha aprendido algo comigo, especialmente a ser mais tolerante em relação a horários e prazos.

Ao CETEM/MCT pela oportunidade e pelo apoio para a realização deste trabalho.

À Vice-Reitoria para Assuntos Acadêmicos da PUC-Rio pela bolsa de isenção da mensalidade.

À Lusinete Patrício de Araújo, secretária da pós-graduação do DCMM, um verdadeiro antídoto contra a burocracia.

Ao amigo Dr. Guilherme Lúcio Abelha Mota pela revisão do capítulo de Reconhecimento de Padrões e pelas frutíferas discussões acerca do tema.

À Biblioteca Técnica da Bielo-Rússia (http://lib.org.by) por diversas das referências deste trabalho.

A todo pessoal da Biblioteca Setorial do CTC da PUC-Rio pela constante simpatia e presteza no atendimento.

A Adauto José da Silva e Edivaldo da Conceição Silva (Nininho), técnicos do CETEM/MCT, pela ajuda na preparação das amostras.

A Rogério Kwitko Ribeiro, geólogo da CVRD, pelas amostras de Sossego.

A Omar Juarez Soto, ex-colega de DCMM, pela amostra de Yauri Cusco.

Ao meu pai, Pedro, e seu pessoal pela construção e desenho em CAD do porta-amostras de uso misto.

Ao meu filho, Gabriel, que nasceu há poucos dias, por ser um moleque tranqüilo e me deixar concluir este trabalho.

Ao Saddam pela agradável companhia.

Aos amigos do CETEM e do DCMM pelo apoio e incentivo.

Aos garçons e demais pessoas que de alguma forma tenham contribuído para este trabalho. 


\section{Resumo}

Gomes, Otávio da Fonseca Martins. Microscopia Co-Localizada: Novas Possibilidades na Caracterização de Minérios. Rio de Janeiro, 2007, 105p. Tese de Doutorado - Departamento de Ciência dos Materiais e Metalurgia, Pontifícia Universidade Católica do Rio de Janeiro.

A integração do controle por computador de microscópios com a aquisição e análise digital de imagens levou à criação de uma nova área, denominada Microscopia Digital. Além de permitir um certo grau de automação, a Microscopia Digital abriu possibilidades realmente novas para a caracterização microestrutural. Uma destas novas e promissoras possibilidades é a Microscopia Co-localizada, que junta diversos tipos de informação, obtidas a partir de diferentes técnicas de microscopia. No presente trabalho foi desenvolvida e implementada uma metodologia de Microscopia Co-localizada que combina imagens de Microscopia Óptica de Luz Refletida (MO) e de Microscopia Eletrônica de Varredura (MEV). Esta metodologia envolve desde a aquisição das imagens nos microscópios até a análise das fases presentes através de técnicas de Reconhecimento de Padrões. Um procedimento automático de registro entre os dois tipos de imagens foi desenvolvido, permitindo o ajuste de magnificação, translação, rotação, tamanho de pixel e distorções locais. Desta forma, imagens de MO e de MEV de uma dada amostra podem ser combinadas precisamente. A metodologia foi testada com diversas amostras minerais, visando a discriminação de fases que são indistinguíveis por MO ou MEV. A Microscopia Co-localizada MOMEV foi empregada em uma rotina para a caracterização de amostras de minério de ferro e os resultados obtidos foram comparados com os da análise tradicional ao MEV.

\section{Palavras-chave}

Microscopia digital; caracterização de minérios; análise de imagens; classificação; minério de ferro; minério de cobre. 


\section{Abstract}

Gomes, Otávio da Fonseca Martins. Co-Site Microscopy: New Possibilities in the Ore Characterization. Rio de Janeiro, 2007, 105p. D.Sc. Thesis Department of Materials Science and Metallurgy, Catholic University of Rio de Janeiro.

Computer-controlled microscopes with digital image acquisition and analysis led to the creation of a new field, called Digital Microscopy. Digital Microscopy not only allows a certain degree of automation but also has brought new possibilities to microstructural characterization. One of these new and promising possibilities is CoSite Microscopy, that links different kinds of information, obtained from different microscopy techniques. The present work presents the development and implementation of a Co-Site Microscopy methodology that combines images obtained by Reflected Light Microscopy (RLM) and Scanning Electron Microscopy (SEM). This methodology involves the whole sequence, from image acquisition at the microscopes to the analysis of the phases using Pattern Recognition techniques. An automatic registration procedure for the two kinds of images was developed, allowing the adjustment of magnification, translation, rotation, and pixel size, and the correction of local distortions. The methodology was tested with several mineral samples, aiming at the discrimination of phases that are not distinguishable with either RLM or SEM. The RLM-SEM Co-Site Microscopy technique was employed in the characterization of iron ore samples and the obtained results were compared to the traditional analysis by SEM.

\section{Keywords}

Digital microscopy; ore characterization; image analysis; classification; iron ore; copper ore. 


\section{Sumário}

1 Introdução

2 Microscopia Digital 18

2.1. Imagem digital 19

2.2. Microscopia Óptica de Luz Refletida 25

2.2.1. Autofoco 25

2.2.2. Plano de foco 28

2.2.3. Correção de defeitos da aquisição de imagens digitais 28

2.2.4. Varredura da amostra e aquisição automática de imagens 33

2.3. Microscopia Eletrônica de Varredura 34

2.4. Microscopia Co-localizada $\quad 35$

$\begin{array}{ll}\text { 2.4.1. Registro } & 37\end{array}$

3 Processamento e Análise Digital de Imagens $\quad 41$

4 Reconhecimento de Padrões 46

4.1. Classificação supervisionada 48

4.2. Classificação supervisionada de pixels 52

4.3. Escolha das características 56

4.4. Classificador 58

5 Microscopia Co-Localizada MO-MEV 63

5.1. Motivação 63

5.2. Metodologia 65

5.2.1. Aquisição de imagens ao microscópio óptico 65

5.2.2. Aquisição de imagens ao MEV 68

5.2.3. Registro 69

5.2.3.1. Registro - Estágio 1

5.2.3.2. Registro - Estágio 2

5.2.3.3. Registro - Estágio 3 
5.2.4. Análise de imagens

5.3. Resultados e Discussão 76

5.3.1. Registro 76

5.3.2. Delineação 80

5.3.3. Análise de imagens 80

6 Microscopia Co-Localizada Aplicada à Caracterização de um Minério de Ferro 86

6.1. Introdução 86

6.2. Procedimento Experimental 88

6.2.1. Resina X Quartzo 88

6.2.2. Microscopia Co-Localizada MO-MEV 89

6.3. Resultados e Discussão 89

6.3.1. Resina X Quartzo 89

6.3.2. Microscopia Co-Localizada MO-MEV 90

7 Conclusão 95

8 Referências bibliográficas $\quad 97$

$\begin{array}{ll}\text { Apêndice A } & 104\end{array}$ 


\section{Lista de figuras}

Figura 1 - Imagem digital. $\quad 19$

Figura 2 - Resolução e quantização. $\quad 20$

Figura 3 - Histograma de níveis de intensidade. 22

Figura 4 - Imagem RGB e suas componentes. 23

Figura 5 - Uma imagem RGB e seu histograma bidimensional RG. $\quad 24$

Figura 6 - Convergência do autofoco. 27

Figura 7 - Imagens inicial e final do procedimento de autofoco. 27

Figura 8 - Não-uniformidade na iluminação. $\quad 29$

Figura 9 - Diferença entre imagens de um mesmo campo. 29

Figura 10 - Intensidade do pixel central de diversas imagens de um mesmo campo. 30

Figura 11 - Perfis de intensidade dos pixels da linha horizontal central da: (a) imagem original; (b) imagem amostrada pela média de oito imagens; e (c) imagem amostrada pela média de oito imagens e com o fundo corrigido. 33

Figura 12 - Transformações em registro 2D/2D. 38

Figura 13 - Seqüência padrão de PADI. 42

Figura 14 - Delineação. $\quad 43$

Figura 15 - Segmentação. $\quad 44$

Figura 16 - Pós-processamento. $\quad 45$

Figura 17 - Reconhecimento e classificação. $\quad 45$

Figura 18 - Espaço de características. $\quad 47$

Figura 19 - Classificação supervisionada. $\quad 48$

Figura 20 - Classificação não-supervisionada. 48

Figura 21 - Exemplo de classificação supervisionada (classificação de frutas). $\quad 50$

Figura 22 - Imagens de referência da norma ISO-945. 50

Figura 23 - Classificação de partículas de grafita em ferro fundido. 51

Figura 24 - Imagem RGB e sua representação no espaço de características RG. 
Figura 25 - Segmentação por classificação de função

paralelepípedo. 55

Figura 26 - Redução de dimensionalidade. 58

Figura 27 - Distância euclidiana x Mahalanobis. 60

Figura 28 - Pirita, pentlandita e calcopirita: (a) imagem de MO; (b) imagem de MEV (BSE). $\quad 64$

Figura 29 - Bornita e esfalerita: (a) imagem de MO; (b) imagem de MEV (BSE). $\quad 65$

Figura 30 - Interface da função LMDcaptura2. 66

Figura 31 - Porta-amostras de uso misto. 68

Figura 32 - Imagens, de um mesmo campo, adquiridas: (a) ao MO;

(b) ao MEV.

Figura 33 - Obtenção dos pontos de controle: (a) imagem de MO; (b) imagem de MEV.

Figura 34 - Pontos de controle. 73

Figura 35 - Imagens após o primeiro estágio do registro. 74

Figura 36 - Imagens após o segundo estágio do registro.

Figura 37 - Resultado final do registro.

Figura 38 - Avaliação do registro (amostra Yauri Cusco). 77

Figura 39 - Campo com menor pico de correlação cruzada normalizada.

Figura 40 - Defeitos devidos a diferenças entre as modalidades de microscopia: as setas brancas apontam lascas e a seta azul indica partículas sob a resina (vistas apenas na imagem de $\mathrm{MO}$ ).

Figura 41 - Delineação de uma imagem RGB: (a) imagem original;

(b) imagem delineada em cada componente; (c) imagem delineada com o filtro que considera as distâncias no espaço RGB.

Figura 42 - Tabela de cores que representa as fases da amostra de Yauri Cusco.

Figura 43 - Variação do conjunto de características: (a) imagem de MO; (b) imagem de MEV; (c) imagem resultado da classificação com as 4 características originais; (d) imagem resultado da classificação com 6 características; (e) imagem resultado da classificação com 10 
características; (f) imagem resultado da classificação com todas as 14 características do sistema expandido.

Figura 44 - Erros de segmentação nas interfaces: as setas apontam regiões erradamente atribuídas à fase covelita.

Figura 45 - Calcopirita e pentlandita: (a) imagem de MO; (b) imagem de MEV; (c) imagem segmentada.

Figura 46 - Bornita e esfalerita: (a) imagem de MO; (b) imagem de MEV; (c) imagem segmentada.

Figura 47 - Fases do minério de ferro em uma: (a) imagem de $\mathrm{MO}$;

(b) imagem de MEV (BSE).

Figura 48 - Resina X Quartzo: (a) imagem de MO de um campo da amostra -149+105 $\mu \mathrm{m}$; (b) histograma da imagem exposta em (a); (c) imagem do mesmo campo recoberto com carbono evaporado; (d) histograma da imagem mostrada em (c).

Figura 49 - Fração de fase de: (a) resina epóxi; (b) quartzo.

Figura 50 - Fração de goethita.

Figura 51 - Segmentação errônea de goethita no MO-MEV-Class: (a) imagem de $\mathrm{MO}$; (b) imagem de $\mathrm{MEV}$; (c) imagem resultado do $\mathrm{MO}$ Class; (d) imagem resultado do MEV-Limiar; (e) imagem resultado do MO-MEV-Class; (f) tabela de cores.

Figura 52 - Segmentação errônea de magnetita e hematita no MO-

Class: (a) imagem de $\mathrm{MO}$; (b) imagem de MEV; (c) imagem resultado do MO-Class; (d) imagem resultado do MEV-Limiar; (e) imagem resultado do MO-MEV-Class; (f) tabela de cores.

Figura 53 - Método híbrido (MEV-Limiar + MO-MEV-Class): (a) imagem de MO; (b) imagem de MEV; (c) imagem resultado do método híbrido (MEV-Limiar + MO-MEV-Class); (d) tabela de cores. 


\section{Lista de tabelas}

Tabela 1 - Amostras.

63

Tabela 2 - Alguns minerais presentes nos minérios de cobre.

Tabela 3 - Correspondência das magnificações. 71

Tabela 4 - Goethita, hematita e magnetita. 86

Tabela 5 - Amostras do minério de ferro 88 